CARNETS OE Carnets de géographes

GÉOGRAPHES.

\title{
Pour une géographie des plaisirs urbains
}

Mathilde Beaufils et Luc Illien

\section{(2) OpenEdition}

Journals

Édition électronique

URL : http://journals.openedition.org/cdg/817

DOI : $10.4000 /$ cdg. 817

ISSN : 2107-7266

Éditeur

UMR 245 - CESSMA

Référence électronique

Mathilde Beaufils et Luc Illien, "Pour une géographie des plaisirs urbains », Carnets de géographes [En ligne], 9 | 2016, mis en ligne le 20 décembre 2016, consulté le 30 avril 2019. URL : http:// journals.openedition.org/cdg/817; DOI : 10.4000/cdg.817

Ce document a été généré automatiquement le 30 avril 2019

\section{(c) (i) (9)}

La revue Carnets de géographes est mise à disposition selon les termes de la Licence Creative Commons Attribution - Pas d'Utilisation Commerciale - Pas de Modification 4.0 International. 


\title{
Pour une géographie des plaisirs urbains
}

\author{
Mathilde Beaufils et Luc Illien
}

\section{NOTE DE L'AUTEUR}

Dans le cadre du séminaire « Géographie des émotions » organisé par Pauline Guinard et Bénédicte Tratnjek à l'Ecole normale supérieure de Paris, Charlotte Ruggeri et Frédérique Célérier sont venues présenter le 12 mars 2015 la revue en ligne Urbanités ${ }^{1}$. Elles sont respectivement directrice de la publication et rédactrice en chef des chroniques et dossiers régionaux. Dans le cadre de ce séminaire, elles sont venues parler plus spécifiquement du numéro d'avril 2014 portant sur les plaisirs urbains.

Au moment de cette présentation, Charlotte Ruggeri était docteure en géographie, professeure en classes préparatoires et venait tout juste de soutenir sa thèse en mars 2015 sur la ligne à grande vitesse californienne. Frédérique Célérier était ATER et doctorante en géographie à l'Université Bordeaux-Montaigne. Sa thèse concerne les territoires du vin biologique en France.

\section{La revue Urbanités : présentation du support}

1 La revue Urbanités, c'est le projet de quatre doctorants de l'ENS de Lyon: ils sont géographes et ont pour horizon commun, malgré leur différente spécialité, la ville et l'urbain. Ce projet est né du constat qu'il n'y avait très peu de revues consacrées aux études urbaines. À travers un nouvel angle et un nouveau support, ils ont abordé ce projet éditorial avec enthousiasme et une démarche particulière: proposer des numéros thématiques avec des approches transversales mêlant sciences humaines et sociales, arts, littérature, photographie... Pour Frédérique Célérier, l'idée était de favoriser l'échange et la rencontre entre plusieurs disciplines et d'en faire la ligne directrice de la revue. 
2 Urbanité sa fait son chemin depuis sa première mise en ligne en novembre 2012. Le choix du support internet s'est implémenté au processus de création puisqu'il a permis une plus grande liberté de formats. La ligne éditoriale avait pour volonté de laisser la place à l'image, aux photographies, aux cartes, à des vidéos... Les articles sont courts et visuels sans être superficiels ou trop vulgarisés : la rigueur scientifique n'est pas négligée tout en privilégiant de vraies approches empiriques. Libre et gratuite d'accès, Urbanités vise un public varié. Depuis peu, une association soutient financièrement la sortie de chaque numéro ainsi que l'ensemble des activités réalisées dans le cadre de la revue.

3 Urbanités présente d'autres catégories comme des chroniques qui ne s'inscrivent pas forcément dans le thème des articles du numéro. On retrouve aussi le "Lu", catégorie qui recense des comptes-rendusd'ouvrages divers qui collent à l'actualité éditoriale. Plus visuel, la catégorie "Vu" nous propose des compte-rendus d'exposition et des photos par le biais du portfolio. Ce portfolio contient des légendes explicatives (exemple de ceux réalisés à Détroit ou Belfast). Enfin la rubrique "Entendu" nous permet de lire des comptes-rendus de conférences, des entretiens avec des chercheurs mais aussi diverses personnes.

\section{La genèse du numéro sur les plaisirs urbains}

4 Dès l'été 2013, le comité de rédaction avait la thématique en tête. Le terme plaisir n'est cependant pas apparu tout de suite, le comité de rédaction voulait avant tout valoriser un thème plus positif que les urbanités souterraines ou encore les villes en crise (objets des deux premiers numéros alors en ligne). Cette décision a aussi été le fruit de rencontres opportunes: rencontres au FIG (Festival international de géographie), discussions sur le thème du corps dans la ville, de la sexualité en ville ... Ils ont tout de suite envisagé des contributions potentielles et des personnes à contacter. Dès lors, certains mots clés ont émergé des réflexions collectives autour du thème de l'érotisation de la ville et la ville du jeu : casino, cinéma, springbreak, parade urbaine, consommation de drogue, ou encore "lèche vitrines". Elle cite aussi les plaisirs sensoriels, la rencontre amoureuse, la rencontre sexuelle mais aussi toutes autres formes de consommations : boire, manger... Par le biais de cette réflexion, cette idée de repenser le terme de géographie grâce à l'entrée du plaisir, un nouvel enjeu de recherche s'est imposé de lui-même : Comment le plaisir, une émotion qui relève de l'intime et de l'individu, s'inscrit-elle alors dans le collectif?

5 Dans le cadre de ce séminaire, Frédérique Célérier a pour volonté de montrer comment on passe de la fixation d'une ligne éditoriale au numéro final et ainsi d'observer les écarts entre les attentes et le produit fini. Elle nous présente donc les grandes lignes directrices de l'appel à contributions :

6 - La réflexion sur la spatialité des plaisirs urbains : Comment les villes sont associées aux plaisirs collectifs dans l'imaginaire? Quels sont les impacts de la localisation de ces villes? Est-ce que les lieux en marge peuvent contribuer à faire émerger ces villes de plaisir? Dans cet optique, l'étude de la proximité des aéroports et des frontières dans des villes de transit est-elle intéressante?

7 - Le thème du plaisir comme vecteur d'attractivité. 
8 - La place du plaisir : est-il caché ou circonscrit à certains espaces ? Faut-il le dérober à la vue de tous? Quels sont les lieux dédiés au plaisir? Existe-il un détournement des lieux par une pratique elle-même?

9 - La place de la normativité et la transgression: certains plaisirs sont-ils autorisés ou interdits ? Le plaisir est-il toujours associé à la débauche et à la décadence ? Doit-on se limiter à parler des plaisirs reconnus par la société ? N'est-ce pas concevoir le plaisir uniquement de manière normative? A l'inverse, le plaisir n'est-il pas dans la discrétion ? Ne le retrouve-t-on pas particulièrement dans les interstices de l'espace urbain?

10 L'appel à contributions a été lancé en novembre 2013 via les réseaux sociaux et les réseaux académiques et personnels. L'association a aussi contacté des auteurs comme Claire Brisson qui travaille sur le corps de la plage à Rio ${ }^{2}$ ainsi qu'un collectif de photographes Périscope ${ }^{3}$ qui a réalisé le portfolio sur l'image du plaisir en ville pour ce numéro. Frédérique Célérier nous explique que le délai choisi a été court entre l'appel à contributions et la date de sortie (avril 2014) pour des raisons d'allègement des processus de publications.

11 Vient ensuite le processus de sélection : le comité de rédaction a reçu plus de trente-cinq propositions pour ce numéro. Ses membres se sont alors réunis et ont choisi selon la qualité de rédaction et si le fond de l'article correspond à l'appel lancé. Deux difficultés majeures pour le comité dé rédaction sont de vérifier que les articles ne sont pas de simples recyclages d'articles plus anciens, et de choisir entre les doublons lorsque les terrains se ressemblent. Quinze propositions ont finalement été retenues.

12 Dans le cas de ce numéro ${ }^{4}$ les différents thèmesabordés correspondent vraiment à l'appel à contributions. La revueprésente une réelle diversité des plaisirs en villes avec une approche pluridisciplinaire. Cette approche est d'autant plus intéressante qu'elle traite d'un sujet moderne et donc relativement peu exploré. On retrouve aussi un aspect d'ambivalence dans ces plaisirs : l'idée de la régulation de ces plaisirs ou de la subversion, ce qui montre le caractère foisonnant de ces derniers, est également traitée.

\section{Les différents thèmes abordés dans le numéro}

13 Après nous avoir parlé de la genèse, Frédérique Célérier nous présente succinctement les différents thèmes abordés dans ce numéro et nous cite quelques articles.

\section{Premier thème : Ville de la tentation}

14 Ce premier thème est axé sur les dispositifs et les aménagements mis en place pour les plaisirs. Cette orientation de recherche présente un paradoxe : la ville est le lieu de la régulation qui obéit à des lois d'urbanisme, un lieu utilitaire où l'on verrouille les corps. Finalement le plaisir , qui renvoie à la liberté ou à l'oisiveté ne serait-il pas l'inverse ? En partant de cette hypothèse, ces articles jouent sur ce paradoxe. Dans cette même rubrique, on s'intéresse à la localisation de ces plaisirs, sont-ils en situés en marge de l'espace public urbain fonctionnel ? Une dernière question concernera le plaisir comme élément principal de l'attractivité d'une ville.

15 Frédérique nous cite l'article de Claire Brisson "Sea, sand and sun: Rio s'éclate". Cet article traite de la plage et de ses pratiques à Rio, en mélangeant une approche anthropologique et géographique. Quelle est la place de la nudité et la liberté associée à 
ces pratiques au cœur et à la marge de Rio ? Un deuxième article "Urbanisme et plaisirs : mise en dialogue au travers de la constitution du mont Royal (Montréal)" de Pierre Bussière, s'interroge sur le rapport entre urbanisme et plaisirs. La ville est le haut lieu de la régulation. Le plaisir quantà lui est lié à la surprise, à l'imprévu. Comment alors créer du plaisir en ville avec l'urbanisme? Quels sont les éléments, les dispositifs qui permettent une jouissance spatiale ? Comment réintroduire de l'intime dans l'espace urbain? Enfin l'article de Charlotte Ruggeri sur Disneyland entre dans ce premier thème.

16 L'intervention de Charlotte Ruggeri est fondée sur son article « Disneyland, un artifice urbain voué au plaisir » publié dans le numéro 3 de la revue Urbanités ${ }^{5}$. Elle présente son article dans une deuxième partie de séminaire pour exposer une conception très particulière du plaisir urbain, celle de Walt Disney et de sa compagnie. Cette conception va à l'encontre du plaisir de la ville tel qu'il peut se définir comme le plaisir de l'imprévu et de la transgression.

\section{Deuxième thème : Jouissance en ville}

17 Cet axe s'est beaucoup penché sur la représentation du plaisir en ville. Un bon nombre de ces articles n'ont pas une approche géographique. On peut citer un article sur la photographie qui présente un compte rendu analytique de deux ouvrages photographiques. Cet article d'Aurélien Cohen ${ }^{6}$ interroge l'importance de la place blanche et de la rue des Lombard. Aurélien Cohen revient sur les différences entre une prostitution qui s'affiche et une prostitution dissimulée dans les impasses. Cette représentation de la ville est alors lascive et charnelle. Le collectif Périscope, nous présente un portfolio autour de l'idée de vitrine, un espace urbain qui est consommé, l'idée que derrière le plaisir, il y a la mélancolie et l'amertume. Toujours avec une

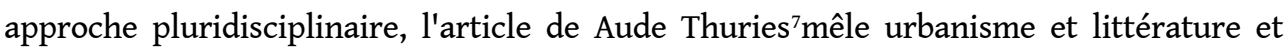
nous montre les représentations du plaisir à travers la comédie. La transgression en ville bien qu'ici virtuelle est abordé par l'article de Axel Scoffier'sur la ville dans les jeux vidéos (GTA V). Le plaisir est alors de retrouver un espace familier dans lequel on peut dépasser les limites de l'urbain. Le dernier article traite du "Parkour"9et est signé de Benoît Tellez. La ville est alors athlétisée, les adhérents de cette pratique ne voient pas la rue comme un "simple piéton"

\section{Troisième thème : plaisir, villes et transgression}

18 Ce thème est le fil directeur du numéro pour Frédérique Célérier. La question de la régulation des plaisirs y est abordée. Un premier article d'Elise Billiardest consacré à la place des plaisirs dans un quartier voué à ces derniers à Malte. Ce quartier est maintenant conquis par les touristes, ce qui entraîne des nuisances pour les riverains. Cela génère un conflit lié à l'appropriation de cet espace. La question concerne alors la moralisation de ce plaisir. Autour de cette même thématique, on retrouve l'article de Louise Dorignon sur la consommation d'alcool et les espaces de musique à Melbourne. Ici, les conflits et les tensions sont nés autour de la régulation de ces pratiques pour des raisons de sécurité. L'alcool est aussi abordé dans l'article de Maximilien Pierre Esse Ndjeng où on s'intéresse à la transformation d'un quartier autour d'une distillerie de bière dans un contexte urbain pas forcément lié aux plaisirs 15 . La consommation de bière a créé là-bas un véritable vecteur d'intégration et de transformation sociale. Plus près de chez nous, 
l'article d'Ulysse Lassaubetraite des pratiques homosexuels dans l'espace urbain de Paris qui ne sont pas du tout acceptées dans ce contexte hétéronormé. Les espaces de ces rencontres amoureuses sont dissimulées, on parle d'interstices publiques. L'article expose les dispositifs régulateurs qui visent à stopper ces pratiques illicites. Enfin, le dernier article traite de la place des "Ciné porn" au Brésil. L'auteur, Joao Pena montre comment ces cinémas, alors lieux importants dans un contexte social dans les années 1970 sont maintenant poussés en périphérie de la ville.

\section{NOTES}

1. http://www.revue-urbanites.fr

2. Brisson Claire."Mise en scène du corps, Expérience de l'espace. Une géographie de la performance de genre sur

les plages de Rio de Janeiro" http://www.theses.fr/s95567.

3. http://www.collectifperiscope.com

4. http://www.revue-urbanites.fr/3-plaisirs-urbains/

5. http://www.revue-urbanites.fr/3-disneyland-un-artifice-urbain-voue-au-plaisir/

6. Cohen, Aurélien. “L'impasse et La Guêpière.” Urbanités, nº 3 (2014).

7. Thuries, Aude. "Dancing in the Street." Urbanités, $\mathrm{n}^{\circ} 3$ (2014)

8. Scoffier, Axel. "L'air de Los Santos rend libre : le plaisir de l'urbain dans GTA V", Urbanités, $\mathrm{n}^{\circ} 3$ (2014)

9. Tellez, Benoit. “Etre Où Ne Pas Être : Poétique Du Parkour”, Urbanités, n 3 (2014).

INDEX

Thèmes : Carnets de lectures

Mots-clés : émotions, urbanité

\section{AUTEURS}

\section{MATHILDE BEAUFILS}

ENS ULM Géographie

mathilde.beaufils@ens.fr

LUC ILLIEN

ENS ULM Géosciences

luc.illien@ens.fr 